\title{
Tailoring highly conductive graphene nanoribbons from small polycyclic aromatic hydrocarbons: a computational study
}

\author{
A Bilić ${ }^{1}$ and S Sanvito ${ }^{2}$ \\ ${ }^{1}$ CSIRO Mathematics, Informatics and Statistics, Private Bag 33, Clayton South 3169 VIC, Australia \\ ${ }^{2}$ School of Physics and CRANN, Trinity College, Dublin 2, Ireland
}

\begin{abstract}
Pyrene, the smallest two-dimensional mesh of aromatic rings, with various terminal thiol substitutions, has been considered as a potential molecular interconnect. Charge transport through two terminal devices has been modeled using density functional theory (with and without self interaction correction) and the non-equilibrium Green's function method. A tetrasubstituted pyrene, with dual thiol terminal groups at opposite ends, has been identified as an excellent candidate, owing to its high conductance, virtually independent of bias voltage. The two possible extensions of its motif generate two series of graphene nanoribbons, with zigzag and armchair edges and with semimetallic and semiconducting electron band structure, respectively. The effects related to the wire length and the bias voltage on the charge transport have been investigated for both sets. The conductance of the nanoribbons with a zigzag edge does not show either length or voltage dependence, owing to an almost perfect electron transmission with a continuum of conducting channels. In contrast, for the armchair nanoribbons a slow exponential attenuation of the conductance with the length has been found, due to their semiconducting nature.
\end{abstract}

\section{Introduction}

In the search for suitable interconnects for nanodevices and molecular circuitry, since the first successful isolation of graphene in the laboratory [1], an interest has arisen in the meshes of this material [2]. Their organic analogs, polycyclic aromatic hydrocarbons (PAHs) have attracted particular attention because of the possibility to synthesize high molecular weight hexagonal carbon meshes [3]. Despite the high expectations of favorable charge transport through $\mathrm{PAH}$ wires, recent theoretical investigations have generally predicted a rather poor conductance and demonstrated an ineffective use of the conjugated carbon backbone [4-7].
An optimal class of molecular interconnects is expected to provide a high and consistent level of conductance for a length range of tens of nanometers and over a sizable bias region. In contrast, a strong localization regime is typically predicted for the transport even for the short PAHs [4-7]. Recently multiple chemical anchoring groups have been proposed for a more efficient coupling across the entire junction [8]. This simple idea has not only resulted in a dramatically enhanced electronic transport, but also in a much improved mechanical and thermal stability, which is also necessary for usable circuit operation.

The smallest PAH with a 2D hexagonal mesh is pyrene, with four benzene rings in the plane. Previous theoretical 
investigations have unanimously predicted a low conductance for pyrene [4, 5, 7] and an even worse charge transport by its oligomers [6], due to the nonresonant tunneling mechanism and strong localization regime. Here we report computational results on the conductance through pyrene in various orientations with respect to the transport axis and terminal surfaces and with variable number and position of thiol head groups. After the identification of a highly conductive pyrene derivative two series of extended graphene nanoribbons were considered on the basis of this isomer. Both series display a high conductance. Whereas one set demonstrates an exponential attenuation of conductance with nanoribbon length, the other series exhibits a length and bias-independent conductance. Hence, the latter set shows very desirable transport properties as potential interconnects in molecular circuits.

\section{Methods}

The geometries of all the junctions considered here have been fully optimized, via the action of the conjugate gradient procedure, until the forces were smaller $10 \mathrm{meV} \AA^{-1}$. The optimizations were performed using density functional theory (DFT) as implemented in the SIESTA program [9]. The LDA functional [10] was used in both the DFT and transport calculations.

A junction of $\mathrm{Au}(001)$ terminated leads has been utilized to model the two terminal device. The $\mathrm{Au}(001)$ surface was described by a square superstructure of eight gold atoms per layer. The optimized geometries obtained from the SIESTA computations, as well as all the relevant computational parameters, were subsequently employed in electron transport calculations. The latter were conducted using the non-equilibrium Green's function (NEGF) Landauer approach [11] as implemented in the SMEAGOL package [12-14], which is interfaced to SIESTA. Self interaction correction (SIC) has been applied in combination with the LDA to the scattering region of the junction to partly rectify the artifacts arising from the band misalignment between the wire and terminals [15-18]. In the present work this has been done by utilizing the atomic self interaction correction (ASIC) [19, 20]. A value of 1.0 was used for the scaling parameter $\alpha$, amounting to the full ASIC.

\section{Results and discussion}

In addition to the conventional 1,6-pyrene dithiol (1,6-PDT), with thiol groups on the long axis, two other para isomers of PDT have been considered, 2,7- and 3,8-PDT and a meta-substituted 2,8-PDT. In addition, a pyrene molecule with dual anchoring thiol groups has also been considered as a potential wire. This leads to two additional possibilities: pyrene-1,2,6,7-tetrathiol (PTT) and 2,3,7,8-PTT. Other combinations, such as 1,3,6,8-PTT, are not considered because the additional separation between the thiols would make double attachment to the terminals impossible, while 3,4,8,9-PTT has been treated elsewhere [21] very recently.
Based on previously published results, thiols bind to the low index gold surface most favorably via the hollow and bridge sites [22, 23]. Accordingly, two junction conformations have been considered for the 1,6-PDT wire: adsorbed on hollow and bridge sites, both in the upright orientation. Clearly, the other three PDT isomers cannot be adsorbed in the upright orientation and the same goes for the two PTT wires. Instead, these were initially positioned in the junction with the $\mathrm{S}$ atoms above either a hollow or a bridge site, with the pyrene plane perpendicular to the terminal surfaces. The fully optimized geometries of the six junctions are illustrated in figure 1 .

The corresponding $I-V$ characteristics and zero-bias transmission spectra are shown in figure 2 . The relatively low conductances calculated for the four PDT junctions are in agreement with the previously predicted transport properties of pyrene and its oligomers between gold electrodes [4-7]. The associated transmission spectra display familiar features: a broad peak below the Fermi energy and another peak above, well separated by a sizable gap. The two peaks representing the most important occupied and unoccupied electron transmission channels are characteristic for aromatic wires (see, e.g., figure 5 in [24]), and they typically originate from the frontier molecular orbital, highest occupied (HOMO) and lowest unoccupied (LUMO), levels. In the case of the 1,6-PDT junctions, however, the symmetry prevents these from establishing an effective coupling with the electrodes. The most relevant frontier MOs with an out-of-plane $\pi$ character, from the third highest occupied (HOMO-2) to the third lowest unoccupied (LUMO +2 ), are illustrated in figure $\mathrm{S} 1$ in the supplementary data (available at stacks. iop.org/JPhysCM/25/275301/mmedia) ${ }^{3}$. Both the HOMO and LUMO are antisymmetric with respect to the mirror plane $\sigma_{\mathrm{v}}$ perpendicular to the molecular plane. Consequently, their orbital weights on the central column of atoms are zero. Hence, the single thiol groups, also situated on the axis, can at best provide a weak, through space, coupling of these MOs to the contacts. Thus, these two most important MOs do not make a substantial contribution to the transmission. In contrast, the second HOMO (HOMO-1), which is nearly degenerate with the HOMO, and the second LUMO (LUMO+1), are both symmetric, they exhibit an excellent conjugation across the whole backbone and provide the dominant contribution to the conductance of 1,6-PDT. Other frontier MOs suffer from either a poor conjugation on the $\mathrm{C}$ backbone (e.g. HOMO-2), or zero orbital weights on terminal thiols, or both (e.g. LUMO+2). The 2,7-PDT isomer exhibits a much better conjugation for all the frontier MOs, in particular the HOMO and LUMO, as shown in figure S2 (available at stacks.iop.org/JPhysCM/25/ $275301 /$ mmedia). The MO densities of 3,8-PDT, shown in figure S3 (available at stacks.iop.org/JPhysCM/25/275301/ mmedia), generally display very similar characteristics to

${ }^{3}$ See supplementary data (available at stacks.iop.org/JPhysCM/25/275301/ mmedia) at URL http://iopscience.iop.org for the plots of the orbital density of the frontier MOs of 1,6-PDT, 2,7-PDT, 3,8-PDT, 2,8-PDT, 1,2,6,7-PTT and $2,3,7,8$-PTT. The electronic states in the -1.5 to $2.0 \mathrm{eV}$ interval for the $2,3,7,8$-PTT junction, whose energies are labeled with blue triangles in the bottom panel of figure 2 are also shown therein. 

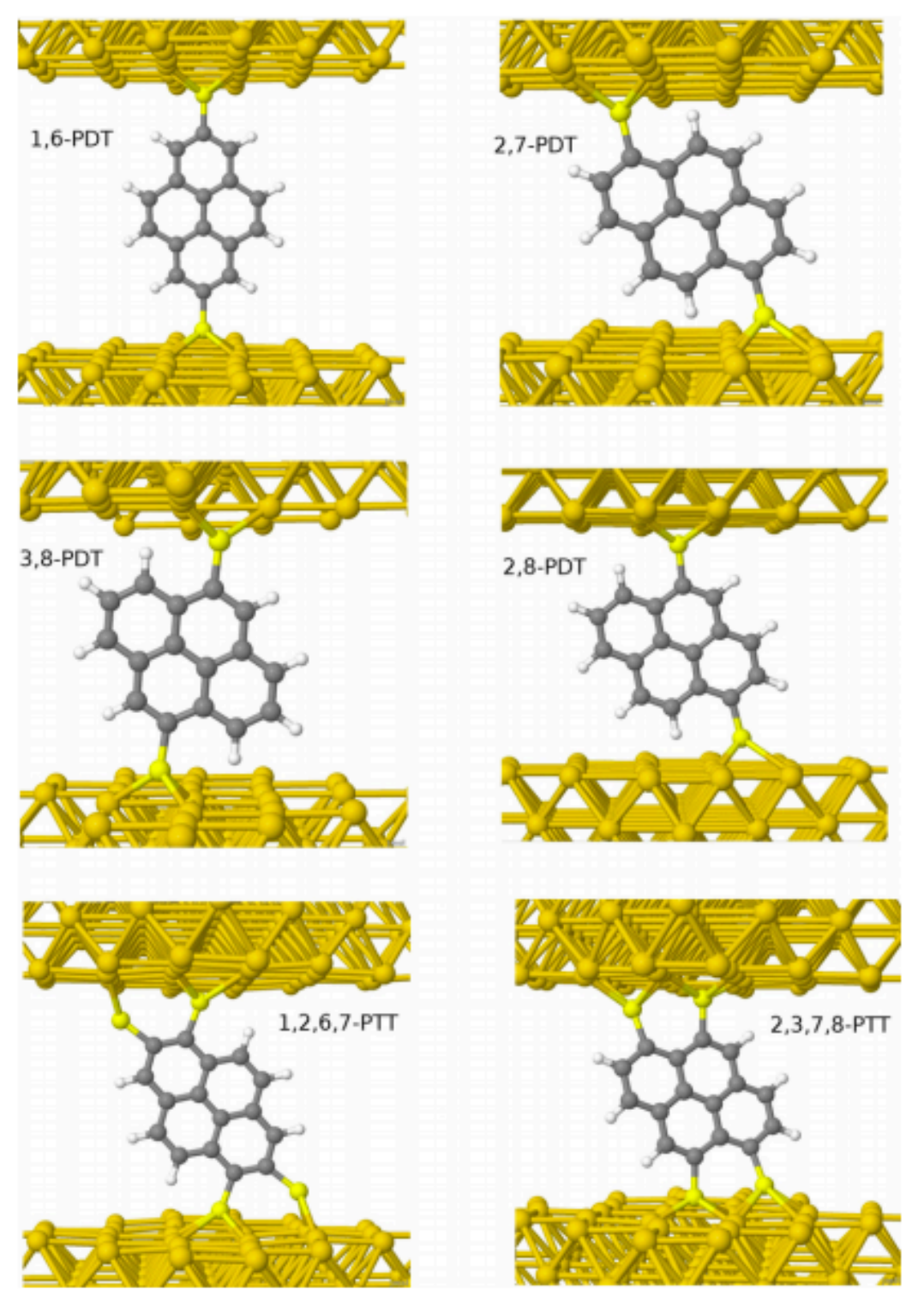

Figure 1. The optimized geometries of the PDT and PTT derivatives between the Au(001) terminals.

Table 1. Calculated HOMO and LUMO energy levels and the energy gap between them for the five pyrene derivatives, given in $\mathrm{eV}$.

\begin{tabular}{lcccccc}
\hline & $1,6-P D T$ & $2,7-P D T$ & $3,8-P D T$ & $2,8-P D T$ & $1,2,6,7-P T T$ & $2,3,7,8-P T T$ \\
\hline LUMO & -3.069 & -2.992 & -3.032 & -2.992 & -3.058 & -2.868 \\
HOMO & -5.388 & -5.087 & -5.287 & -5.063 & -5.127 & -4.701 \\
Gap & 2.319 & 2.095 & 2.255 & 2.071 & 2.069 & 1.833 \\
\hline
\end{tabular}

those of 2,7-PDT. Consequently, their transmission spectra in figure 2 show the same features, although with more subdued amplitudes in the case of 3,8-PDT. The first occupied peak below the Fermi level is particularly attenuated for 3,8-PDT, which is the consequence of the $0.2 \mathrm{eV}$ lower HOMO energy than that of 2,7-PDT, given in table 1 . The predicted transport properties and frontier MO density and energy levels of the meta 2,8-PDT isomer bear even more resemblance to those of 2,7-PDT (MO densities are shown in figure S4 available at stacks.iop.org/JPhysCM/25/275301/mmedia). In general, it is evident that all four PDT isomers exhibit similar conductance channels and, unsurprisingly, similar current magnitudes are predicted in the low bias regime.

Combining the individual benefits of 1,6-PDT and 2,7-PDT, as in the case of 1,2,6,7-PTT, yields somewhat improved characteristics, in particular for the HOMO, which exhibits a high orbital density on all the terminal thiols, shown in figure S5 (available at stacks.iop.org/JPhysCM/ $25 / 275301 /$ mmedia). As a consequence, the occupied peak just below the Fermi level in the 1,2,6,7-PTT transmission 

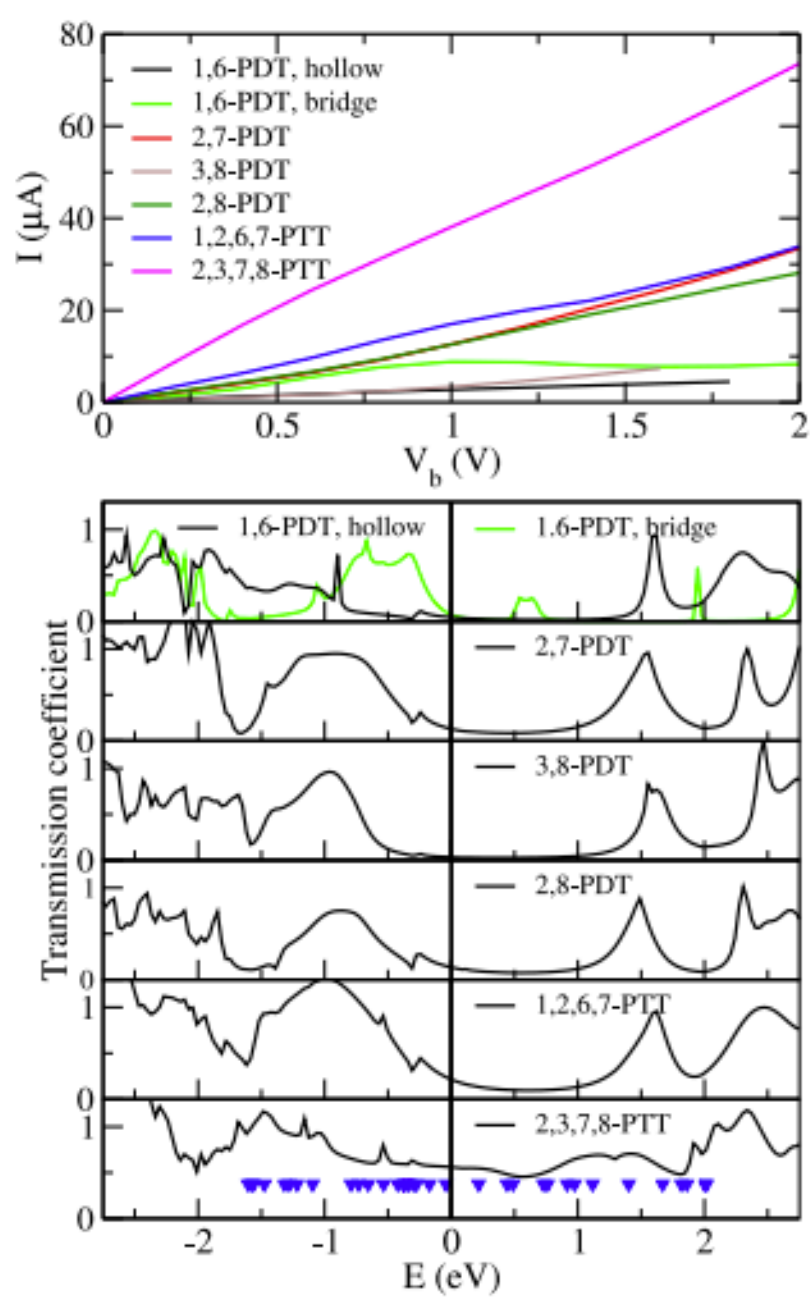

Figure 2. Upper panel: current versus bias voltage for the six pyrene junctions as calculated from the LDA (ASIC uncorrected). Lower panels: the zero-voltage transmission probability across these junctions. The blue triangles in the bottom panel indicate the $\Gamma$ point energies of the electronic states that give rise to the transmission continuum. The energy is given relative to the Fermi level.

spectrum is substantially amplified, resulting in a higher low bias conductance than any of the PDT isomers. However, as in the case of 3,4,8,9-PTT [21], the benefits of the reduced contact resistance are rather limited, because the two thiol groups at each terminal are connected to the same $\mathrm{C}$ ring. This is a poor design since it does not qualitatively change the charge flow pathway.

To open up multiple transport pathways across the entire junction, a single anchor group per terminal $\mathrm{C}$ ring is the most effective mode of wire attachment. This is the motivation behind the 2,3,7,8-PTT isomer design. All the frontier MOs, shown in figure S6 (available at stacks.iop.org/JPhysCM/25/ $275301 /$ mmedia), exhibit a high orbital density on thiol links (although not on both S atoms for HOMO-2 and HOMO-1) and a good conjugation across the $\mathrm{C}$ backbone. The nature of the LUMO +2 also indicates the importance of the in-plane $\pi$ MOs for the conductance of this isomer. As an additional benefit, the gap between the HOMO and LUMO energies, given in table 1 , is $0.2-0.5 \mathrm{eV}$ smaller for 2,3,7,8-PTT than for the other pyrene derivatives. Contrary to the spectra of the other four derivatives, the transmission coefficient of the 2,3,7,8-PTT, in the bottom panel of figure 2, displays qualitatively very different characteristics. The conductance gap, prominent in all the other spectra, has completely disappeared and so have the distinct peaks. In contrast, this spectrum exhibits a flat continuum of highly conducting channels in the relevant energy interval of $\pm 1 \mathrm{eV}$ from the charge neutrality point. Consequently, the associated $I-V$ characteristics demonstrates a very high conductance, nearly independent of the bias voltage. The transport properties of 2,3,7,8-PTT suggests that this junction utilizes numerous electronic states whose energies are close to the Fermi level. Given the flat transmission spectrum in this energy range, it is not possible to single out particularly prominent states that give rise to highly conductive transport channels. Instead, one can identify the important MOs by considering orbital density distribution (at the zone center) of the electronic states whose energy is near the Fermi level and with a strong orbital conjugation across the entire junction, i.e. the gold electrodes, the thiol head groups, and the carbon backbone. This analysis reveals about two dozen of relevant electronic states in the $\pm 1 \mathrm{eV}$ interval, most of which have an out-of-plane $\pi$ character. The $\Gamma$-point energies of the electronic states that give rise to the conducting channels are indicated by blue triangles in the bottom panel of figure 2 . The corresponding orbital plots of these are shown in figure S7 (available at stacks.iop.org/JPhysCM/25/275301/mmedia). The large number of these states, with strong coupling between gold and PTT, manifested by the high degree of dispersion away from the zone center, give rise to the continuous flat transmission spectrum.

The potential of pyrene for the high, bias-independent conductance has, until the present work, remained elusive [4-7]. The significance of the present findings is to show how even the smallest PAH with a two-dimensional hexagonal mesh, pyrene, still inherits the highly desirable charge transport properties of graphene. A further consideration of the structural relationship between pyrene and extended graphene meshes provides probably the clearest explanation of the remarkably good conductance of 2,3,7,8-PTT. The 2,3,7,8-PTT motif can be extended into two series of graphene nanoribbons (GNRs). The first way generates a class of zigzag GNRs conventionally labeled 3-zGNR [25], while the alternative produces an armchair, 6-aGNR, series. Both 3-zGNR and 6-aGNR are illustrated in figure 3. In line with previous predictions from both tight binding $[2,25]$ and first principles $[26,27]$ calculations, the computed band structure in figure 3 indicates that the $3-z G N R$ is a quasi semimetal with a near zero band gap, while the 6-aGNR is a semiconductor with a sizable direct band gap. However, for finite length nanoribbons both series will exhibit substantial HOMO-LUMO gaps, as shown in figure 4. Hence, it is interesting to evaluate the conductance of both the series as a function of length. The results may also provide useful information on what series would be more suitable as molecular interconnects in nanosize devices.

The $I-V$ characteristics and zero-bias transmission coefficients for wires from the 3-zGNR class with four (4R), 

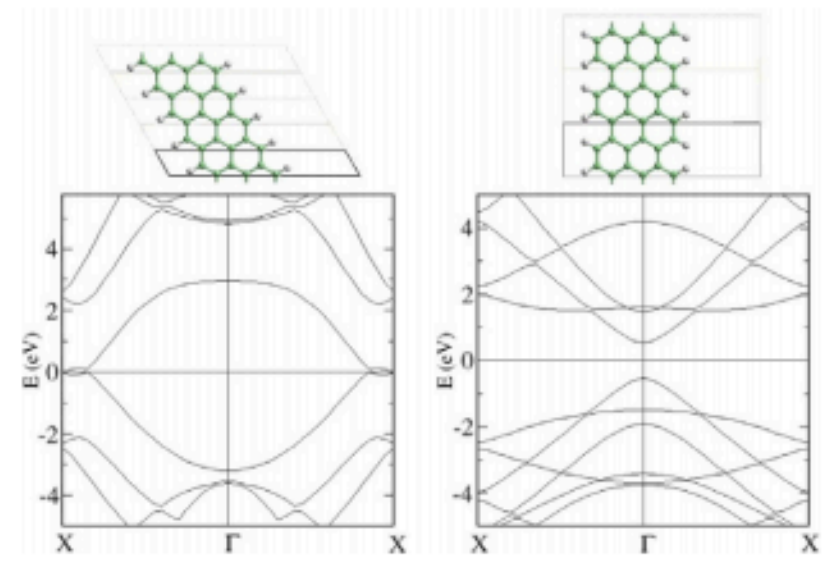

Figure 3. Energy bands of the frontier electronic states of the 3-zGNR (left panel) and 6-aGNR (right panel). The energy is given relative to the Fermi level. The unit cells are shown above the plots.

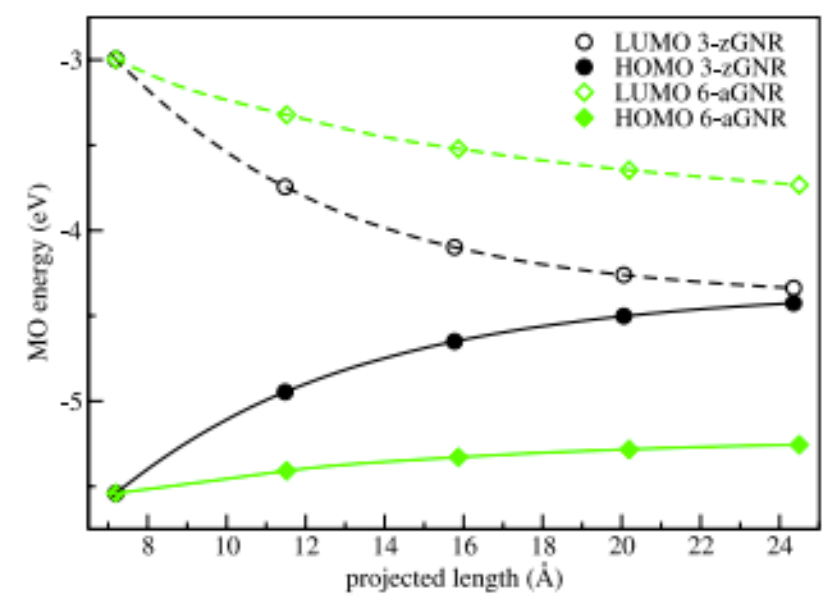

Figure 4. The HOMO and LUMO energy levels of the 3-zGNR and 6-aGNR series as calculated from the LDA (ASIC uncorrected). The lines are merely guides to the eye. The horizontal axis is the projection of the wire length on the transport axis.

eight (8R), twelve (12R), sixteen (16R) and twenty (20R) rings are shown in figure 5. Several favorable observations can be made. Firstly, very high current levels are predicted for all the wires, with no visible variation with the length. Secondly, all the $I-V$ characteristics are linear, resulting in a high conductance which is independent of either length or bias voltage across the series. Hence, the contacts are providing the key contribution to the total resistance. The transmission spectra for $4 \mathrm{R}$ and $8 \mathrm{R}$ exhibit a continuum of channels with high transmission probabilities over a large energy range. In contrast, for the longer members, 12-20R, the spectra show an increasingly rich peak structure, reflecting the importance of the delocalized out-of-plane $\pi$ electronic states for the long range transport. The bottom panel in figure 5 demonstrates a high degree of correlation between the projected density of states (PDOS, out-of-plane contribution) on two representative $\mathrm{C}$ atoms, situated along the nanoribbon edge and in the central row, and the transmission spectrum. The two sharp peaks in the 12-20R spectra, on each side of the Fermi energy, arise from the HOMO and LUMO
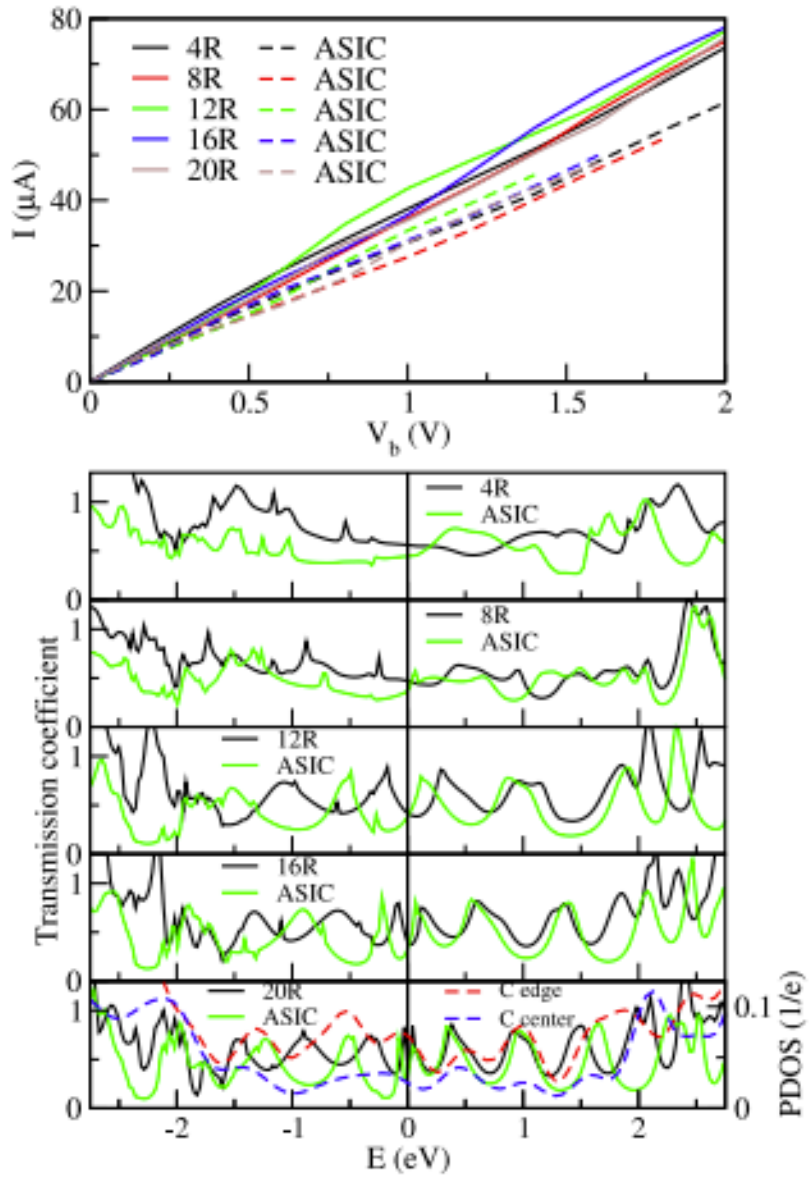

Figure 5. Upper panel: current versus bias voltage for the $4 R, 8 R$, $12 \mathrm{R}, 16 \mathrm{R}$ and $20 \mathrm{R}$ nanoribbons from the 3-zGNR series, calculated from the LDA and ASIC corrected. Lower panels: the zero-voltage transmission probability across these junctions. The energy is given relative to the Fermi level. The out-of-plane orbital contribution to the partial density of states (PDOS) in the $20 \mathrm{R}$ junction from a C atom on the edge and in the central row is also shown in the bottom panel.

and make the key contribution to the low bias conductance. Finally, the inclusion of the ASIC results in a slight reduction of the conductance for all wires. Qualitatively though, the ASIC corrected results are in excellent agreement with their uncorrected counterparts.

The $I-V$ characteristics and zero-bias transmission coefficients for wires from the 6-aGNR class with four (4R), eight (8R), twelve (12R), sixteen (16R) and twenty (20R) rings are shown in figure 6 . In contrast to their 3-zGNR counterparts, an attenuation of the current with length is evident across the entire bias range. An inspection of the low bias conductance, shown in figure 7 , reveals an exponential dependence on nanoribbon length. From a least squares fitting procedure a rather low attenuation factor $\beta$ of $0.125 \AA^{-1}$ is obtained for this series. As expected, the application of the ASIC produces an increasingly wider energy gap between the occupied and unoccupied channels in the transmission spectra. As a consequence, the relatively high uncorrected conductance of the shorter members $4-12 R$ is substantially reduced. For $16 \mathrm{R}$ and $20 \mathrm{R}$, which show sizable gaps even 

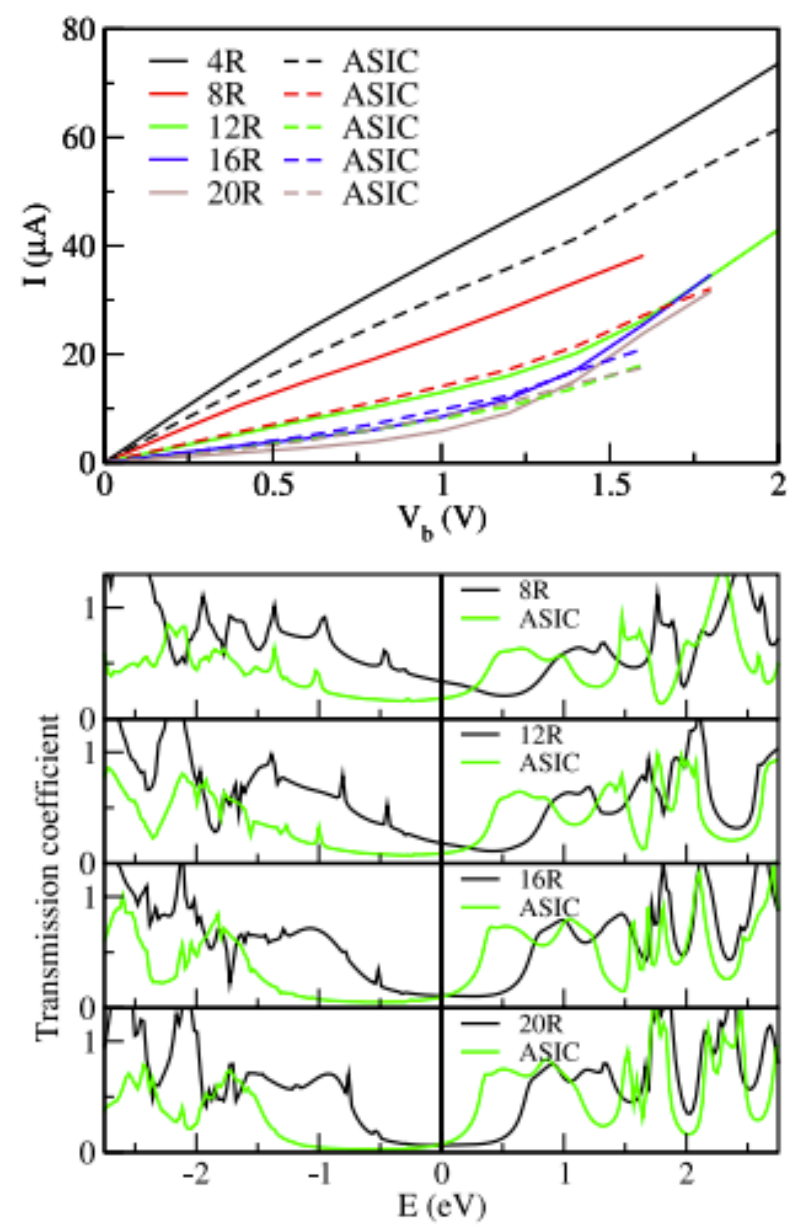

Figure 6. Upper panel: current versus bias voltage for the 4R, 8R, 12R, 16R 20R nanoribbons from the 6-aGNR series, calculated from the LDA and ASIC corrected. Lower panels: the zero-voltage transmission probability across these junctions. The energy is given relative to the Fermi level.

in the uncorrected spectra, the effects of the ASIC appear marginal.

Most commonly the conjugated aromatic wires exhibit an exponential decay, $\exp (-\beta L)$ of the conductance with molecule length $(L)[6,28-30]$, which is consistent with the nonresonant tunneling mechanism or superexchange [31]. This behavior, predicted for 6-aGNRs in the present work, can be understood in terms of Simmons' model [28, 31]. In the molecular junctions for which the frontier MO energy levels remain sufficiently separated from the Fermi energy the factor $\beta$ itself will not exhibit a large variation with length. This is exactly the case with the HOMO and LUMO levels of the 6-aGNRs series, as shown in figure 4. In contrast, as the same plot shows, the variation of these levels with molecule length for 3-zGNRs is too rapid to make a common factor $\beta$ possible for this series. Hence, this simple relation no longer applies. Instead, the detrimental effects of nanoribbon length are fully compensated by the narrowing energy gap between the Fermi and frontier MO levels. Furthermore, as the spectrum in the bottom panel of figure 5 shows, for $20 \mathrm{R}$ (and, presumably, longer members beyond 20R) the

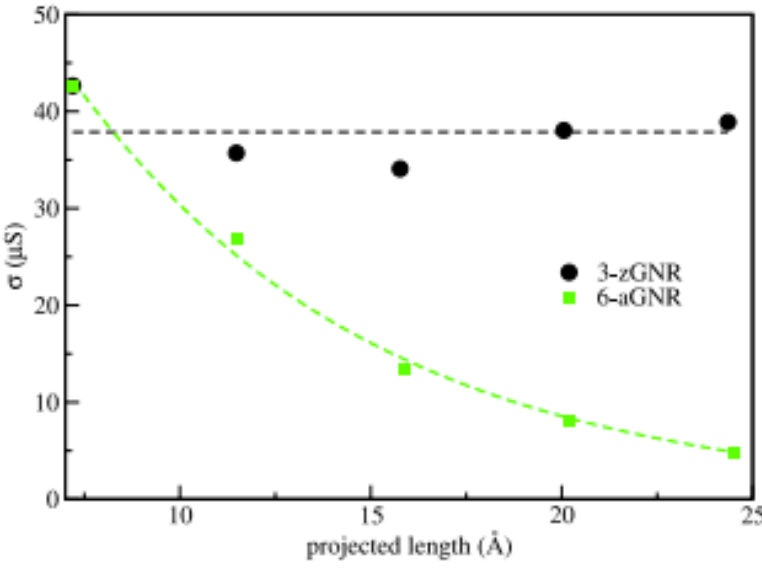

Figure 7. The low bias conductance of the $3-\mathrm{zGNR}$ and 6-aGNR series as calculated from the LDA (ASIC uncorrected). The lines are least squares fits of the points to constant and exponential functions, respectively. The horizontal axis shows the projection of the wire length on the transport axis.

low bias charge flow is practically resonant. The two peaks nearly coinciding with the Fermi level represent the highly conducting graphene electronic states with linear dispersion. Hence, the length independent trend is expected to continue for even longer members of this series. A similar length independent conductance, which is a signature of ballistic transport, has been observed in carbon nanotubes [32, 33].

\section{Conclusions}

The conductance of pyrene in various orientations with respect to the gold electrodes has been modeled using the NEGF approach combined with DFT calculations. The 2,3,7,8-thiolated form of pyrene has been found to have excellent charge transport properties, manifested by the high conductance virtually independent of bias voltage. Thus, it has been shown that the smallest of the 2D PAHs has the potential, previously overlooked, for use as a molecular wire. Based on the 2,3,7,8-PTT motif two series of short GNRs, 3-zGNR with zigzag and 6-aGNR with armchair edges, have been inspected as nanocircuit interconnects. The 3-zGNR set exhibits a conductance practically independent of the nanoribbon length and the bias voltage, making it an almost ideal wire. The semiconducting nature of the 6-aGNRs, combined with the nonresonant tunneling mechanism of charge transport, causes an exponential attenuation of conductance with the length.

The present findings indicate the importance of the multiple terminal groups for an optimal use of available electron transport channels and multiple conjugated pathways across the junction. The ballistic nature of charge transport through the 3-zGNRs shows that the contact points with gold are the principal source of resistance. The proposed dual terminal points not only substantially reduce the resistance, but in addition, provide a much more effective coupling to the gold electrodes and they open up the multiple pathways of charge flow across the entire junction. Our results demonstrate 
that with these PAHs and short GNRs can realize their full potential for charge transport applications.

\section{Acknowledgments}

This work was supported by the CSIRO Advanced Materials Transformational Capability Platform. A B thanks CSIRO for support through the Julius Career Award. The use of the NCI National Facility supercomputers at the ANU is gratefully acknowledged. The SMEAGOL project is sponsored by Science Foundation of Ireland (grant no. 07/IN/I945), by KAUST (FIC/2010/08) and by CRANN.

\section{References}

[1] Geim A K and Novoselov K S 2007 The rise of graphene Nature Mater: 6183

[2] Dubois S M-M, Zanolli Z, Declerc X and Charlier J-C 2009 Electronic properties and quantum transport in graphene-based nanostructures Eur. Phys. J. B 721

[3] Wu J, Pisula W and Müllen K 2007 Graphenes as potential material for electronics Chem. Rev. 107718

[4] Cohen R, Stokbro K, Martin J M L and Ratner M A 2007 Charge transport in conjugated aromatic molecular junctions: molecular conjugation and molecule-electrode coupling J. Phys. Chem. C 11114893

[5] Liu H, Ni W, Zhao J, Wang N, Guo Y, Taketsugu T, Kiguchi M and Murakoshi K 2009 Nonequilibrium greens function study on the electronic structure and transportation behavior of the conjugated molecular junction: terminal connections and intramolecular connections J. Chem. Phys. 130244501

[6] Liu H, Yu C, Gao N and Zhao J 2010 The diversity of electron-transport behaviors of molecular junctions: correlation with the electron-transport pathway ChemPhysChem 111895

[7] Li X, Staykov A and Yoshizawa K 2010 Orbital views of the electron transport through polycyclic aromatic hydrocarbons with different molecular sizes and edge type structures J. Phys. Chem. C 1149997

[8] Martin-Lasanta A, Miguel D, Garcia T, Lopez-Villanueva J A, Rodriguez-Bolivar S, Gomez-Campos F M, Bunuel E, Cardenas D J, Alvarez de Cienfugos L and Cuerva J M 2012 Influence of the number of anchoring groups on the electronic and mechanical properties of benzene-, anthracene- and pentacene-based molecular devices ChemPhysChem 13860

[9] Soler J M, Artacho E, Gale J D, García A, Junquera J, Ordejón P and Sánchez-Portal D 2002 Density-functional method for very large system with LCAO basis sets J. Phys.: Condens. Matter 142745

[10] Ceperley D M and Alder B J 1980 Ground state of the electron gas by a stochastic method Phys. Rev. Lett. 45566

[11] Damle P S, Ghosh A W and Datta S 2003 Nanoscale device modeling Molecular Nanoelectronics ed M A Reed and T Lee (Los Angeles: American Scientific Publishers) p 115

[12] Rocha A R, Garcia-Suarez V M, Bailey S W, Lambert C J, Ferrer J and Sanvito S 2005 Towards molecular spintronics Nature Mater. 4335

[13] Rocha A R, Garcia-Suarez V M, Bailey S W, Lambert C J, Ferrer J and Sanvito S 2006 Spin and molecular electronics in atomically-generated orbital landscapes Phys. Rev. B 73085414

[14] Rungger I and Sanvito S 2008 Algorithm for the construction of self-energies for electronic transport calculations based on singularity elimination and singular value decomposition Phys. Rev. B 78035407
[15] Toher C, Filippetti A, Sanvito S and Burke K 2005 Self-interaction errors in density-functional calculations of electronic transport Phys. Rev. Lett. 95146402

[16] Reimers J R, Solomon G C, Gagliardi A, Bilic A, Hush N S, Frauenheim T, Di Carlo A and Pecchia A 2007 The greens function density functional tight-binding (GDFTB) method for molecular electronic conduction J. Phys. Chem. A 1115692

[17] Toher C and Sanvito S 2007 Efficient atomic self-interaction correction scheme for nonequilibrium quantum transport Phys. Rev. Lett. 99056801

[18] Toher C and Sanvito S 2008 Effects of self-interaction corrections on the transport properties of phenyl-based molecular junctions Phys. Rev. B 77155402

[19] Pemmaraju C D, Archer T, Sánchez-Portal D and Sanvito S 2007 Atomic-orbital-based approximate self-interaction correction scheme for molecules and solids Phys. Rev. B 75045101

[20] Filippetti A, Pemmaraju C D, Sanvito S, Delugas P, Puggioni D and Fiorentini V 2011 Variational pseudo-self-interaction-corrected density functional approach to the $a b$ initio description of correlated solids and molecules Phys. Rev. B 84195127

[21] Bilic A and Sanvito S 2013 Anomalous length dependence of the conductance of graphene nanoribbons with zigzag edges J. Chem. Phys. 138014704

[22] Bilic A, Reimers J R and Hush N S 2005 The structure, energetics, and nature of the chemical bonding of phenylthiol adsorbed on the $\mathrm{Au}(111)$ surface: implications for density-functional calculations of molecular-electronic conduction J. Chem. Phys. 122094708

[23] Bilic A, Gale J D and Sanvito S 2011 From fused aromatics to graphene-like nanoribbons: the effects of multiple terminal groups, length and symmetric pathways on charge transport Phys. Rev. B 84205436 (See errata in [34])

[24] Biliê A, Crljen Ž, Gumhalter B, Gale J D, Rungger I and Sanvito S 2010 Conductance of a phenylene-vinylene molecular wire: contact gap and tilt angle dependence Phys. Rev. B 81155101

[25] Nakada K, Fujita M, Dresselhaus G and Dresselhaus M S 1996 Edge state in graphene ribbons: nanometer size effect and shape edge dependence Phys. Rev. B 5417954

[26] Soon Y-W, Cohen M L and Louie S G 2006 Energy gaps in graphene nanoribbons Phys. Rev. Lett. 97216803

[27] Soon Y-W, Cohen M L and Louie S G 2006 Half-metallic graphene nanoribbons Nature 444347

[28] Venkataraman L, Klare J E, Tam I W, Nuckolls C, Hybertsen M S and Steigerwald M L 2006 Single-molecule circuits with well-defined molecular conductance Nano Lett. 6458

[29] Quek S Y, Choi H J, Louie S G and Neaton J B 2009 Length dependence of conductance in aromatic single-molecule junctions: experiment and theory Nano Lett. 93949

[30] Venkataraman L, Klare J E, Nuckolls C, Hybertsen M S and Steigerwald M L 2006 Dependence of single-molecule junctions conductance on molecular conformation Nature $\mathbf{4 4 2} 904$

[31] McCreery R L 2004 Molecular electronic junctions Chem. Mater. 164477

[32] Frank S, Poncharal P, Wang Z L and de Heer W A 1998 Carbon nanotube quantum resistors Science $2801744-6$

[33] Park J-Y, Rosenblatt S, Yaish Y, Sazonova V, Üstünel H, Braig S, Arias T A, Brouwer P W and McEuen P L 2004 Electron-phonon scattering in metallic single-walled carbon nanotubes Nano Lett. 4 517-20

[34] Bilic A, Gale J D and Sanvito S 2012 Erratum: from fused aromatics to graphene-like nanoribbons: the effects of multiple terminal groups, length and symmetric pathways on charge transport [Phys. Rev. B 84, 205436 (2011)] Phys. Rev. B 86039905 